(2) Open Access Full Text Article

\title{
Positive predictive value of the infant respiratory distress syndrome diagnosis in the Danish National Patient Registry
}

This article was published in the following Dove Press journal:

Clinical Epidemiology

10 August 2013

Number of times this article has been viewed

\section{Sandra Kruchov Thygesen \\ Morten Olsen \\ Christian Fynbo \\ Christiansen}

Department of Clinical Epidemiology, Aarhus University Hospital,

Aarhus, Denmark
Correspondence: Sandra Kruchov Thygesen

Department of Clinical Epidemiology, Aarhus University Hospital, Olof Palmes Allé 43-45, 8200 Aarhus N, Denmark

Tel +4587168063

Fax +4587167215

Email st@dce.au.dk
Background: Infant respiratory distress syndrome (IRDS) is the most common respiratory disease in preterm infants, and is associated with considerable morbidity and mortality. Valid data on IRDS are important in clinical epidemiological research.

Objectives: The objective of this study was to estimate the positive predictive value (PPV) of the IRDS diagnosis registered in the population-based Danish National Patient Registry according to the International Classification of Diseases, 8th and 10th revisions.

Methods: Between January 1, 1977 and December 31, 2008, we randomly selected three patients per year, 96 in total, who were registered with an IRDS diagnosis in the Danish National Patient Registry and living in the northern part of Denmark. Data on the infants included information on the presence of predefined clinical symptoms. We defined IRDS as the presence of at least two of four clinical symptoms (tachypnea, retractions or nasal flaring, grunting, and central cyanosis), which had to be present for more than 30 minutes. Using medical record review as the reference standard, we computed the positive predictive value of the registered IRDS diagnosis including 95\% confidence intervals (CIs).

Results: We located the medical record for 90 of the 96 patients (94\%), and found an overall PPV of the IRDS diagnosis of $81 \%$ (95\% CI 72\%-88\%). This did not vary substantially between primary and secondary diagnoses. The PPV was higher, at 89\% (95\% CI 80\%-95\%), for preterm infants born before 37 weeks of gestation.

Conclusion: The PPV of the IRDS diagnosis in the Danish National Patient Registry is reasonable when compared with symptoms described in the corresponding medical records. The Danish National Patient Registry is a useful data source for studies of IRDS, particularly if restricted to preterm infants. Nonetheless, the potential impact of misclassification of the IRDS diagnosis must be considered.

Keywords: epidemiology, data quality, validity, positive predictive value, hospital diagnosis, respiratory distress syndrome

\section{Introduction}

Infant respiratory distress syndrome (IRDS) is the most common respiratory disease in preterm infants, and leads to substantial morbidity and mortality. ${ }^{1,2}$

IRDS is caused by lung immaturity and usually develops within minutes of birth. It is defined by tachypnea, retractions or nasal flaring, grunting respiration, and possibly central cyanosis. ${ }^{3}$ It occurs in approximately $0.3 \%-1.2 \%$ of live-born infants. ${ }^{4-6}$ However, the prevalence of IRDS increases with decreasing gestational age. ${ }^{4,7}$ Previous studies have found a prevalence of approximately $90 \%$ in premature infants born in gestational week $28 .^{8}$ 
Valid data on IRDS are important for clinical epidemiological research. If the coding is accurate, the Danish medical registries provide excellent data to study the long-term prognosis of IRDS, as the registries comprise more than 30 years of medical observations. ${ }^{9}, 10$

To our knowledge, no study has examined the validity of the IRDS diagnosis in administrative registries. We therefore conducted the present study with the objective of estimating the positive predictive value (PPV) of the IRDS diagnosis recorded in the population-based Danish National Patient Registry (DNPR) according to the International Classification of Diseases (ICD), 8th and 10th revisions, using medical records as reference standard.

\section{Materials and methods Population}

Based on the DNPR, we identified patients diagnosed with IRDS from January 1, 1977 to December 31, 2008 in the northern part of Denmark (corresponding to the former North Jutland County). This part of Denmark has approximately 500,000 inhabitants, equivalent to approximately $11 \%$ of the total Danish population. The entire Danish population is provided with unrestricted tax-supported health care.

\section{The Danish National Patient Registry}

The DNPR includes data on all non-psychiatric hospital admissions in the country since 1977 and outpatient clinic and emergency room visits since 1995. Data include the patients' civil registration number, which is a unique personal identification number assigned to all Danish residents, date of admission and discharge, surgical procedure(s) performed, one primary diagnosis and up to 19 secondary diagnoses coded by the discharging physician according to the ICD-8 until the end of 1993 and subsequently the ICD-10. The primary diagnosis code registered is the main reason for the hospital contact.

Among all patients with a primary or secondary IRDS diagnosis, we randomly selected three IRDS patients for each calendar year, 96 in total, between 1977 and 2008. The IRDS hospital admissions were identified based on the ICD-8 diagnosis code 776.19 (idiopathic respiratory distress syndrome or hyaline membrane disease) and the ICD-10 diagnosis code P22.0 (idiopathic respiratory distress syndrome).

\section{Medical record review}

The medical records of the identified IRDS patients were reviewed and data were entered in EpiData (EpiData
Association, Odense, Denmark) by a physician (SKT). Where there was doubt with regard to interpretation of the medical record, another physician (CFC) was consulted. Data entered included presence of predefined clinical symptoms and X-ray findings. We also noted gender, gestational age, treatment with continuous positive airway pressure (CPAP), and whether the IRDS diagnosis was mentioned explicitly in the medical record.

In the primary analysis, we defined IRDS as the presence of at least two of the four clinical symptoms (tachypnea, retractions or nasal flaring, grunting, and central cyanosis), which had to be present for more than 30 minutes. Tachypnea was defined as 60 or more breaths per minute.

In an additional analysis, we defined IRDS as two or more clinical symptoms together with a positive X-ray finding, defined as reticulogranular ground-glass appearance with air bronchograms. If no information was available on whether or not an X-ray had been taken, or if the radiologist had explicitly ruled out signs of IRDS, we classified the individual as not having IRDS. For descriptive purposes, we abstracted data on CPAP treatment, but only if provided by pediatric departments and for more than 30 minutes. Thus, brief CPAP treatment given immediately after birth was not included. Furthermore, we noted if the IRDS diagnosis was mentioned in the medical record as a confirmed diagnosis.

\section{Statistical analysis}

We used the medical records as the reference standard when computing the PPV of the IRDS diagnosis. The PPV was defined as the proportion of patients registered with an IRDS diagnosis in the DNPR that was confirmed by medical record review. Thus, the numerator was the number of confirmed IRDS cases according to the medical records, and the denominator was the selected number of patients registered with an IRDS diagnosis in the DNPR. The $95 \%$ confidence intervals (CIs) were computed using Jeffrey's method. ${ }^{11}$

We stratified the analyses by primary and secondary diagnoses, by ICD-8 (1977-1993) and ICD-10 (1994-2008) periods, and by gestational age (gestational week $<28$, 28-31, 32-36, and $\geq 37$ ). The study was approved by the Danish Data Protection Agency.

\section{Results}

For 90 of the 96 (94\%) selected patients with an IRDS diagnosis in the DNPR, we were able to find the corresponding medical record, of which 52 (58\%) were for males and $38(42 \%)$ were for females. Gestational age was reported in the medical record for 88 IRDS patients, of whom 65 (74\%) 
were preterm infants, and $23(26 \%)$ were born at term (37 weeks of gestation or later).

From the medical record we were able to confirm 73 of the 90 patients coded with an IRDS diagnosis. This gave us an overall PPV of $81 \%$ (95\% CI 72\%-88\%) (Table 1). In the additional analysis, with IRDS defined as two or more clinical symptoms of IRDS and a confirmed X-ray (52 IRDS patients), the PPV was 58\% (95\% CI 48\%-68\%).

IRDS was registered in the DNPR as the primary diagnosis for 20 (22\%) patients, of which 14 were confirmed by the medical record review, corresponding to a PPV of $70 \%$ (95\% CI 48\%-86\%). Among 70 (78\%) patients registered with IRDS as a secondary diagnosis, 59 were confirmed IRDS patients, corresponding to a PPV of $84 \%(95 \%$ CI 75\%-91\%). We found a PPV of 87\% (95\% CI 75\%-94\%) in the ICD-8 period (1977-1993) and a PPV of 75\% (95\% CI $61 \%-86 \%$ ) in the ICD-10 period (1994-2008). The PPV among males was 75\% (95\% CI 62\%-85\%), while it was $89 \%$ (95\% CI 77\%-96\%) among females. Stratified by gestational age, we found a PPV ranging from 61\% (95\% CI $41 \%-79 \%$ ) in infants born at 37 weeks of gestation or later, to $92 \%$ (95\% CI 77\%-98\%) in infants born between 28 and 31 weeks of gestation (Table 1).

The IRDS diagnosis was explicitly mentioned in $71(79 \%)$ of the 90 medical records, and $82(91 \%)$ of the

Table I Positive predictive value of the infant respiratory distress syndrome diagnosis in the Danish National Patient Registry in 90 patients, 1977-2008

\begin{tabular}{|c|c|c|}
\hline & $\begin{array}{l}\text { Confirmations } \\
(\mathbf{N}=90)\end{array}$ & $\begin{array}{l}\text { PPV \% } \\
(95 \% \mathrm{Cl})\end{array}$ \\
\hline \multicolumn{3}{|l|}{ Diagnostic criteria } \\
\hline Two or more clinical symptoms ${ }^{a}$ & $73 / 90$ & $81(72-88)$ \\
\hline Two or more clinical symptoms & $52 / 90$ & $58(48-68)$ \\
\hline \multicolumn{3}{|l|}{ AND X-ray confirmation } \\
\hline \multicolumn{3}{|l|}{ Type of diagnosis } \\
\hline Primary discharge diagnosis & $14 / 20$ & $70(48-86)$ \\
\hline Secondary discharge diagnosis & $59 / 70$ & $84(75-91)$ \\
\hline \multicolumn{3}{|l|}{ Period according to ICD edition } \\
\hline ICD-8 (1977-I993) & $40 / 46$ & $87(75-94)$ \\
\hline ICD-I0 (I994-2008) & $33 / 44$ & $75(6 \mid-86)$ \\
\hline \multicolumn{3}{|l|}{ Gender } \\
\hline Male & $52 / 90$ & $75(62-85)$ \\
\hline Female & $38 / 90$ & $89(77-96)$ \\
\hline \multicolumn{3}{|c|}{ Gestational age (completed weeks) } \\
\hline 37 weeks of gestation or more & $14 / 23$ & $6 I(4 \mid-79)$ \\
\hline $32-36$ weeks of gestation & $31 / 35$ & $89(75-96)$ \\
\hline $28-31$ weeks of gestation & $23 / 25$ & $92(77-98)$ \\
\hline Less than 28 weeks of gestation & $4 / 5$ & $80(37-98)$ \\
\hline
\end{tabular}

Note: a Clinical symptoms: tachypnea, retractions, grunting, and cyanosis.

Abbreviations: $\mathrm{Cl}$, confidence interval; ICD, International Classification of Diseases, 8 th and 10 th revisions; PPV, positive predictive value. patients with an IRDS diagnosis were treated with CPAP for more than 30 minutes.

\section{Discussion}

In this study, we found reasonable accuracy of the coding for IRDS in the DNPR as confirmed by exact description of the symptoms of IRDS in the medical record. To our knowledge, this is the first study to examine the validity of the DNPR with regards to IRDS. Other studies have estimated the PPV of other neonatal diagnoses in the DNPR, including the diagnoses of congenital cardiac malformations with overlapping time periods, 1994-2002 and 2000-2008..$^{12,13}$ They found overall PPVs of $89 \%$ (95\% CI 86\%-92\%) and $90 \%$ (95\% CI $89 \%-91 \%),{ }^{12,13}$ which is slightly higher than our PPV, probably because IRDS is a syndrome characterized by the co-occurrence of characteristic symptoms.

We found a slightly lower PPV in the ICD-10 period than in the ICD-8 period. However, these estimates were statistically imprecise. The potential decrease in PPV over time may reflect less optimal coding practices in the later period, but may also be explained by better documentation of symptoms in the medical records of the early period. We defined the IRDS diagnosis as the presence of a minimum of two of four clinical symptoms in the medical record. However, these symptoms may not always be described in the medical records of IRDS patients. Our PPV would potentially be an underestimate. The diagnosis of IRDS is complicated as it is a diagnosis of exclusion. If conditions such as infections or congenital heart disease were overlooked by the clinicians, we may have overestimated the PPV of the IRDS diagnosis. We did not include an X-ray finding of IRDS in our main criteria, because $\mathrm{X}$-rays were not routinely performed in patients with mild IRDS. As expected, we found a higher PPV of the IRDS diagnosis among infants born preterm than among infants born at term. This may be due to the higher prevalence of IRDS among children born preterm.

Our study has some limitations that should be considered when interpreting the results. We only examined one region in Denmark; however, we find it reasonable to believe that the results are representative for the entire country owing to the uniform Danish health care system. Further, we were not able to report on sensitivity, ie, the proportion of all patients with IRDS actually registered in the DNPR, as we only included patients with a DNPR diagnosis of IRDS. However, the completeness of the DNPR has previously been estimated to be approximately $90 \% .^{12,14}$ Further, we were not able to blind the IRDS diagnosis for the physician who reviewed the 
medical records; however, this is unlikely to have had any major influence on our findings.

The PPV of the IRDS diagnosis quantified in our study may be applied in sensitivity analyses in future studies, to examine the potential effect of the misclassification on study results. Alternatively, studies should be restricted to infants born before 37 weeks of gestation. Both the primary and the secondary IRDS diagnoses may be used.

\section{Conclusion}

We found a reasonable PPV of $81 \%$ (95\% CI 72\%-88\%) of the IRDS diagnosis in the DNPR, when compared with symptoms described in the infants' medical record. The DNPR is a useful data source for studies of IRDS, particularly if restricted to preterm infants. Nonetheless, the potential impact of misclassification of the IRDS diagnosis should be considered.

\section{Disclosure}

The authors report no conflict of interest in the study.

\section{References}

1. Rodriguez RJ. Management of respiratory distress syndrome: an update. Respir Care. 2003;48(3):279-286.

2. Goldenberg RL, Culhane JF, Iams JD, Romero R. Epidemiology and causes of preterm birth. Lancet. 2008;371(9606):75-84.

3. Rudolph AJ, Smith CA. Idiopathic respiratory distress syndrome of the newborn: an international exploration. J Ped. 1960;57(6):905-921.
4. Koivisto M, Marttila R, Kurkinen-Räty M, et al. Changing incidence and outcome of infants with respiratory distress syndrome in the 1990s: a population-based survey. Acta Paediatr. 2004;93(2):177-184.

5. Rubaltelli FF, Bonafe L, Tangucci M, Spagnolo A, Dani C. Epidemiology of neonatal acute respiratory disorders. A multicenter study on incidence and fatality rates of neonatal acute respiratory disorders according to gestational age, maternal age, pregnancy complications and type of delivery. Italian Group of Neonatal Pneumology. Biol Neonate. 1998;74(1):7-15.

6. Reed DM, Bakketeig LS, Nugent RP. The epidemiology of respiratory distress syndrome in Norway. Am J Epidemiol. 1978;107(4): 299-310.

7. Sweet DG, Carnielli V, Greisen G, et al. European consensus guidelines on the management of neonatal respiratory distress syndrome in preterm infants: 2010 update. Neonatology. 2010;97(4):402-417.

8. Stoll BJ, Hansen NI, Bell EF, et al. Neonatal outcomes of extremely preterm infants from the NICHD Neonatal Research Network. Pediatrics. 2010;126(3):443-456.

9. Sørensen HT. Regional administrative health registries as a resource in clinical epidemiology. A study of options, strengths, limitations and data quality provided with examples of use. Int J Risk Saf Med. 1997; 10(1):1-22.

10. Frank L. Epidemiology. When an entire country is a cohort. Science. 2000;287(5462):2398-2399.

11. Brown LD, Cai TT, DasGupta A. Interval estimation for a binomial proportion. Statist Sci. 2001;16(2):101-133.

12. Jepsen B, Jepsen P, Johnsen SP, Espersen GT, Sørensen HT. Validity of diagnoses of cardiac malformations in a Danish population-based hospital-discharge registry. Int J Risk Saf Med. 2006;18(2):77-81.

13. Agergaard P, Hebert A, Bjerre J, Sørensen KM, Olesen C, Ostergaard JR. Children diagnosed with congenital cardiac malformations at the national university departments of pediatric cardiology: positive predictive values of data in the Danish National Patient Registry. Clin Epidemiol. 2011;3:61-66.

14. Moth G, Vedsted P, Schiøtz PO. National registry diagnoses agree with medical records on hospitalized asthmatic children. Acta Paediatr. 2007;96(10):1470-1473.
Clinical Epidemiology

\section{Publish your work in this journal}

Clinical Epidemiology is an international, peer-reviewed, open access journal focusing on disease and drug epidemiology, identification of risk factors and screening procedures to develop optimal preventative initiatives and programs. Specific topics include: diagnosis, prognosis, treatment, screening, prevention, risk factor modification, systematic

\section{Dovepress}

reviews, risk \& safety of medical interventions, epidemiology \& biostatical methods, evaluation of guidelines, translational medicine, health policies \& economic evaluations. The manuscript management system is completely online and includes a very quick and fair peer-review system, which is all easy to use. 